Revista científica, arbitrada e indizada, bajo la modalidad electrónica.

Recibido: $18 / 06 / 2020$

Aceptado: 13/07/2020

\title{
DECISIONES DE FINANCIAMIENTO EN EMPRESAS FAMILIARES: UNA REVISIÓN Y SUGERENCIAS PARA DESARROLLAR EL CAMPO
}

Financing decisions in family companies: a review and suggestions for developing the field

\author{
Omar El Kadi \\ Financial Advisor \\ Scotiabank Canada \\ Omar.elkadi@scotiabank.com
}

\section{RESUMEN}

Este artículo tiene como propósito examinar a través de un análisis, las decisiones de financiamiento en empresas familiares: Una revisión y sugerencias para desarrollar el campo. Con este fin la investigación estuvo orientada por un enfoque postpositivista, cualitativo, de tipo documental, con diseño bibliográfico, comprendiendo la revisión literaria para conocer el estado del arte de las categorías estudiadas, al igual que el levantamiento de la información obtenida de las bases de datos, revistas científicas, trabajos de grado, repositorios institucionales, así como la identificación de objetivos. Se sustentó en postulados de Cheltenham, Holt, Madison y Kellermanns (2017), Chen, Ding, Wu, y Yang (2016), Chrisman y Holt (2016), King y Peng (2013), Martin y Gómez (2016), El Kadi (2014), entre otros. Los hallazgos demuestran la existencia de gran cantidad de referente teórico sobre la temática, en la cual se desarrolla un aporte en la literatura de financiamiento de empresas familiares y presenta un modelo para guiar la investigación existente y futura mediante la identificación de brechas en las perspectivas teóricas y en elementos específicos del contexto, como la heterogeneidad de la empresa familiar, además de los factores específicos del país.

Palabras clave: Decisiones de financiamiento, Empresas Familiares, Organizaciones financieras, riqueza socioemocional. 


\title{
Revista científica, arbitrada e indizada, bajo la modalidad electrónica.
}

\begin{abstract}
This article aims to examine through an analysis, financing decisions in family companies: a review and suggestions for developing the field. To this end, the research was oriented by a post-positivist focus, qualitative, documentary-type approach, with bibliographic design, comprising literary review to know the state of the art of the categories studied, as well as the survey of the information obtained from databases, scientific journals, degree works, institutional repositories, as well as the identification of objectives. It was based on postulates of Cheltenham, Holt, Madison \& Kellermanns (2017), Chen, Ding, Wu, \& Yang (2016), Chrisman \& Holt (2016), King \& Peng (2013), Martin \& Gomez (2016), El Kadi (2014), among others. The findings demonstrate the existence of a large amount of theoretical reference on the subject, in which a contribution is developed in the literature of financing family companies and presents a model to guide existing and future research by identifying gaps in the theoretical perspectives and context-specific elements, such as the heterogeneity of the family business and the specific factors of the country.
\end{abstract}

Key words: Financing Decisions, Family Enterprises, Financial Organizations, Socioemotional wealth.

\section{INTRODUCCIÓN}

¿Qué sabemos sobre las decisiones de financiación en las empresas familiares?

En esta sección, se analizan los estudios de financiación de empresas familiares en detalle, clasificándolos en tres grupos, sobre la base de la fuente de financiación con la cual tratan:

\section{Deuda}

Cuando las empresas familiares consideran el uso de fuentes externas de financiamiento, el apalancamiento sigue siendo con mucho la opción de financiación preferida para las empresas familiares, los resultados de la investigación dan evidencia de un orden de picoteo en la financiación de las empresas familiares, donde se prefiere la deuda en lugar de nuevo capital cuando se busca financiación externa adicional.

\section{Capital externo}

Otra corriente de investigación se centra en el uso de la equidad externa. Por un lado, varios estudios indican que la participación familiar parece dar lugar a un menor uso de la equidad externa. Por lo general, siguiendo la postura de Chen et al., (2016), la distancia 


\section{Revista científica, arbitrada e indizada, bajo la modalidad electrónica.}

entre las empresas familiares y los inversores externos es bastante grande, principalmente debido a la llamada brecha de empatía entre propietarios e inversores o debido a la retención generalmente preferida del control en lugar del crecimiento y desarrollo de la empresa. Por otro lado, contrariamente a la perspectiva del orden de picoteo, King y Peng (2013), encuentran que, en las industrias caracterizadas por la ciclicaidad, la intensidad del capital y el crecimiento, las grandes empresas familiares cotizadas confían en la financiación de capital antes de la financiación de la deuda para financiar su expansión, principalmente por la fuerte aversión vinculada a la angustia financiera.

Las empresas también pueden adquirir financiación de capital a través de una oferta pública inicial (OPI). Si bien este es un evento importante para todo tipo de empresas, especialmente para las familiares. Después de todo, un cambio en la estructura de propiedad al hacer pública implica un cambio significativo en la gobernanza de las empresas familiares, porque a menudo es la primera vez que los accionistas externos entran en juego. La razón financiera más importante para que las empresas familiares se vuelvan públicas, es la necesidad de recaudar fondos más altos para financiar el crecimiento o reequilibrar el nivel de deuda-Capital.

\section{Ganancias retenidas frente a dividendos}

La teoría del orden de picoteo establece que las empresas prefieren financiar nuevas inversiones primero internamente, a través de ganancias retenidas. Sin embargo, todas las ganancias que se retienen en la empresa no se pueden distribuir como dividendos. Esto ha llevado a los investigadores a examinar esta compensación (ganancias retenidas versus pagos de dividendos) a la que se enfrentan las empresas familiares a la hora de decidir cómo asignar sus ganancias. La mayoría de los artículos estudian la política de dividendos en el contexto de las empresas de titularidad pública, y aproximadamente la mitad de los artículos de dividendos de nuestra revisión de la literatura se centran en las empresas de Asia, donde muchas economías se caracterizan por la considerable propiedad familiar de las corporaciones cotizadas.

Marco para comprender las decisiones de financiación de empresas familiares 
Revista científica, arbitrada e indizada, bajo la modalidad electrónica.

FUENTES DE FINANCIAMIENTO: también están las fuentes alternativas: ejemplo ingreso por rentas, como lo muestra a continuación el gráfico 1.

Grafico 1. Decisiones de Financiamiento en las empresas familiares.

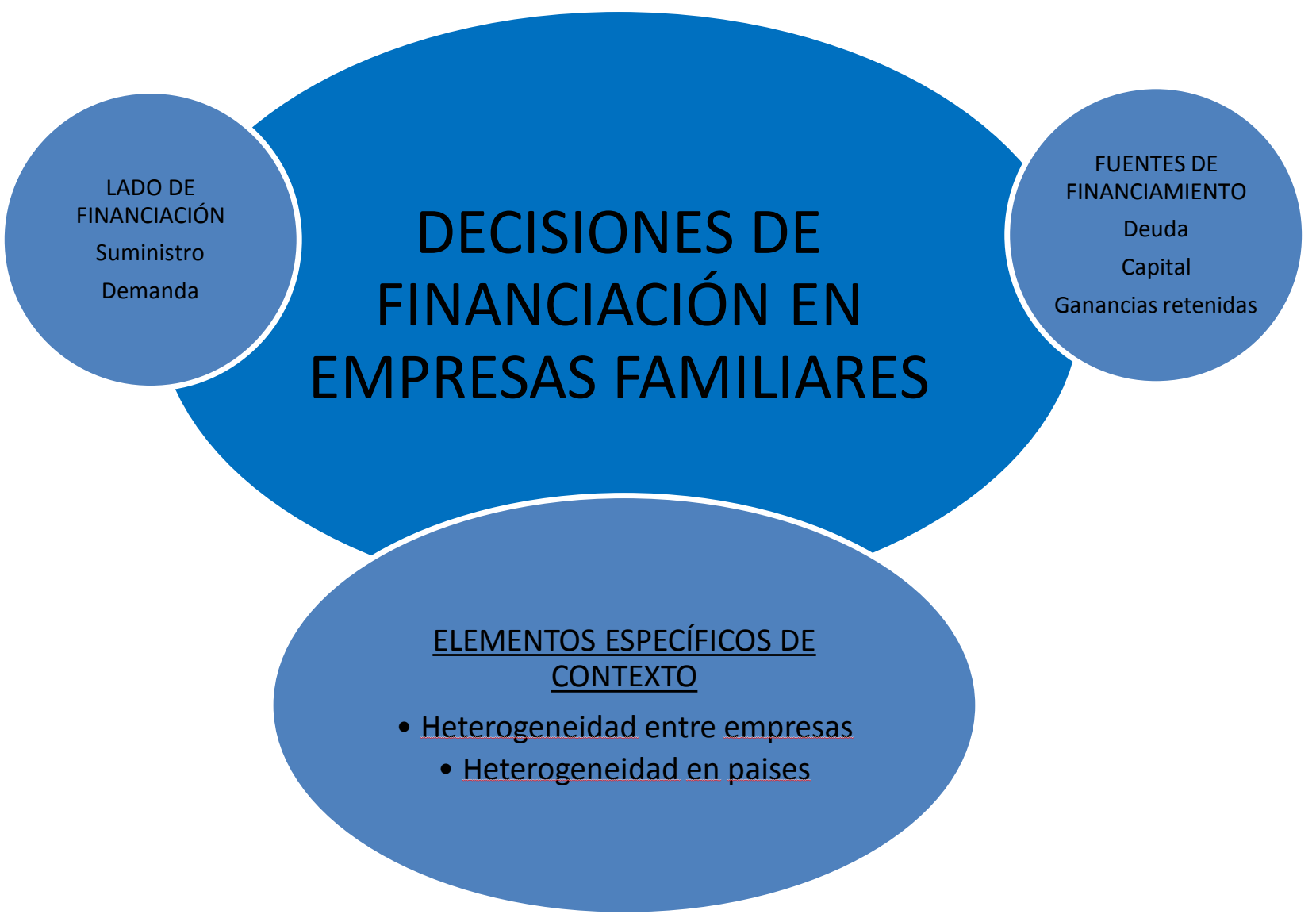

Fuente: Elaboración propia (2020).

\section{Heterogeneidad entre las empresas familiares}

Esta naturaleza heterogénea podría ser capturada teniendo en cuenta los diferentes objetivos, estructuras de gobierno y recursos que tienen. Como estos tres conductores se consideran las fuentes de diferencias de comportamiento entre las empresas familiares y no 


\section{Revista científica, arbitrada e indizada, bajo la modalidad electrónica.}

familiares, proporcionando una excelente base para distinguir entre diferentes tipos de empresas familiares.

Las fuentes de heterogeneidad relacionadas con los objetivos podrían ampliar nuestra visión de las decisiones de financiación de las empresas familiares, por ejemplo, la importancia que los propietarios familiares dan a los objetivos de LA SEW con el fin de explicar el comportamiento de financiación de las empresas familiares. Sin embargo, ninguno de estos estudios ha medido directamente SEW, lo que, esencialmente, hace que sea difícil indicar qué impulsa exactamente el comportamiento de financiación de las empresas familiares. En este sentido, la escala de importancia de la riqueza socioemocional (SEWi), desarrollada recientemente por Debicki et al., (2016) podría ser una medida interesante.

Las fuentes de heterogeneidad relacionadas con la gobernanza surgen de la participación de la familia en la propiedad y la gestión y también pueden conducir a una amplia variedad de resultados. Además, de investigar la diferencia de importancia que se les ha impuesto a las preocupaciones socioemocionales, es importante tener en cuenta la facultad discrecional que tienen los miembros de la familia para perseguir esas preocupaciones. Dado que las decisiones de estructura de capital normalmente están influenciadas o tomadas por el consejo de administración, es probable que el papel y la calidad de este consejo afecten a las decisiones de financiación en las empresas familiares. Martin y Gómez (2016).

Las fuentes de heterogeneidad relacionadas con la fuente que podrían tenerse en cuenta al examinar las decisiones de financiación en las empresas familiares son, por ejemplo: la disponibilidad de activos humanos basados en la familia, la capacidad de profesionalizar la empresa o la presencia de capital de riesgo, que puede influir en los recursos disponibles para la empresa tanto en cantidad como en calidad.

El tamaño de las empresas también puede conducir a la heterogeneidad entre las empresas familiares, ya que podría explicar los objetivos, la gobernanza y los recursos de las empresas familiares, la mayoría de los estudios están orientados hacia grandes empresas públicas, aunque, en realidad, las pequeñas empresas privadas son la norma. Los hallazgos 


\section{Revista científica, arbitrada e indizada, bajo la modalidad electrónica.}

resultantes de muestras de grandes empresas familiares cotizadas no pueden transferirse automáticamente a empresas de propiedad privada que suelen ser de un tamaño mucho menor, por lo que se requieren pruebas más explícitas sobre este efecto de tamaño. Por ejemplo, muchas pequeñas empresas familiares se enfrentarán al analfabetismo financiero, que podría abordarse parcialmente a través del asesoramiento financiero proporcionado por banqueros o contadores.

\section{Heterogeneidad en todos los países}

La mayoría de los artículos empíricos revisados, son estudios de un solo país. Esto es algo sorprendente, dado que varios investigadores han señalado las grandes diferencias que se producen entre los países con respecto a su nivel de protección de los inversores y acreedores, su nivel de desarrollo de los mercados financieros, el grado en que los mercados de capitales están centrados en los bancos, su cultura y su marco jurídico (civil frente al derecho común). Por ejemplo, varios países han introducido normas para corregir el incentivo para asumir una deuda excesiva, como limitar la deducibilidad de los costes de intereses (Francia, Alemania, Hungría, España, Italia) o ampliar la deducibilidad para incluir el coste de la financiación de capital. Estos incentivos podrían influir significativamente en las decisiones sobre la estructura de capital y el pago de dividendos.

Los cambios en la deducibilidad de los costos de intereses, la regulación bancaria (por ejemplo, la aplicación de Basil III) o las perturbaciones económico-financieras. Más concretamente, las normas fiscales podrían disuadir a las empresas familiares a asumir deudas o pagar dividendos en algunos países, mientras que en otros países no lo harán.

\section{EMPRESA FAMILIAR Y ECONOMÍA CANADIENSE}

- Las empresas familiares emplean a seis millones de trabajadores.

- Crea el 70\% de los puestos de trabajo.

- Proporciona el 55\% de todas las donaciones caritativas.

- Las empresas familiares aportan el 60\% del PIB canadiense. 
Revista científica, arbitrada e indizada, bajo la modalidad electrónica.

Gráfico 2. Empresas Familiares y el USO DE JUNTA DIRECTIVA

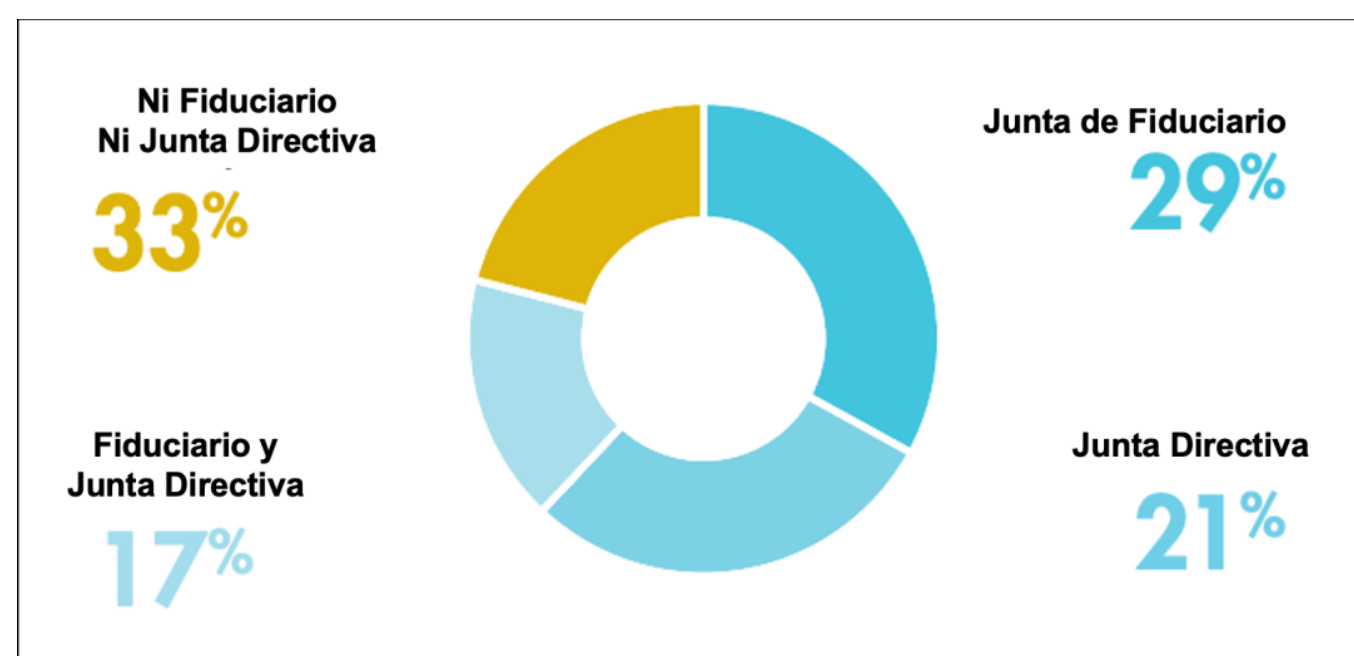

Fuente: Alberta Business Family Institute, junio 2020.

\section{DATOS IMPORTANTES}

Los Consejos Asesores de Consejos Asesores son un portal eficaz para que las empresas familiares accedan a la experiencia, sin la presión de capacitar a los asesores con responsabilidades formales o legales. Los consejos consultivos se adoptan generalmente en la 1a generación, dentro de los primeros 30 años de la empresa. El 63\% de todos los consejos consultivos son pagados, Los consejos consultivos están presididos por miembros de la familia el $44 \%$ del tiempo, mientras que el $34 \%$ de los miembros del consejo asesor son mujeres. Los consejos consultivos tienen un $63 \%$ más de probabilidades de tener funciones combinadas de presidente y CEO

\section{COMPARACIÓN DE GENERACIONES}

$1^{\text {ra }}$ Generación "Los Contructores":

- El negocio está creciendo, pero la base puede necesitar algún trabajo. La primera generación de una empresa familiar está preocupada por el crecimiento. 


\section{Revista científica, arbitrada e indizada, bajo la modalidad electrónica.}

- La familia compromete totalmente el tiempo, dinero y pasión, dejando en Segundo plano los procesos formales.

- Con el fundador prominentemente involucrado en el negocio 93\% - garantizando la creación de valor para la próxima generación -

- Es raro ver sistemas para garantizar un funcionamiento sin problemas para transición generacional

- El Enfoque es CRECIMIENTO y DESEMPEÑO

$2^{\text {da }}$ Generación: "Enfocados en el futuro":

- Una nueva generación significa más miembros de la familia. Más miembros de la familia significa una estructura más formal.

- Una transición exitosa a la segunda generación demuestra los esfuerzos del fundador, que se prepara a Ceder el espacio.

- El enfoque cambia hacia el desarrollo y preparación de la 3ra generación, y formalizando importantes estructuras, prácticas y políticas.

- Las Compañías ya son más grandes, más complejas y tienen un mayor Número de inversionistas con demandas únicas que necesitan ser gestionadas.

- "El fundador no quería una junta, pero la Segunda generación esta más interesada."

- "Los fundadores no creen en el cambio. No compartirán los problemas familiares con extraños "

- Miembros de la familia de segunda generación muestran aumento de preocupación por el desarrollo de la próxima generación, pero solo el $22 \%$ de las empresas tiene un plan de sucesión para los propietarios. Koropp (2013).

$3^{\text {ra }}$ Generación, Permitiendo el ingreso de "Outsiders"

- Para la tercera generación, el fundador generalmente ya no está en la imagen, y la influencia de los Outsider "ajenos a la familia" ha crecido.

- Los negocios de 3ra y 4ta generación los participantes tienen un promedio de 75 años.

- Entre la 2da y 3ra generación, el el enfoque vuelve al desempeño corporativo. 
Revista científica, arbitrada e indizada, bajo la modalidad electrónica.

- La sucesión del CEO según Koropp et al. (2013b), es una de las principales preocupaciones por primera vez, pero todavía se percibe como una realidad distante.

- Mientras tanto, tercera y cuarta generación construyen tablilla de consejeros independientes y reducen la dependencia en consejeros o consultivos informales.

- Si no lo han hecho ya, los procesos de gobernanza se están convirtiendo en un proceso riguroso y formal.

- $\quad$ EL ENFOQUE / PREOCUPACION:

1- Desempeño financiero

2- La sucesión de un CEO

SUPERVIVENCIA DE LA EMPRESA FAMILIAR DURANTE EL COVID-19

- Un dato positivo es que, en muchos casos, las empresas familiares históricamente han sido más resilientes que otras organizaciones en recesiones económicas bruscas e inesperadas.

- Las empresas familiares generalmente han estado en el negocio a través de múltiples recesiones y crisis, lo cual es la base de su enfoque estratégico constante en el largo plazo.

- Al consultar a clientes de empresas familiares durante los declives económicos,

- Hay tres áreas principales que aconsejan para ayudar en las próximas semanas y meses.

\section{Áreas de Enfoque para sobrellevar el declive económico}

- 1- Bienestar del empleado

- Apoyar

- Retener

- Suspender dividendos / compensación

- Si bien es importante permitir la continuidad del negocio, una de las principales prioridades para las empresas familiares durante estos tiempos cambiantes y difíciles es apoyar a sus empleados. 


\section{Revista científica, arbitrada e indizada, bajo la modalidad electrónica.}

- Las empresas familiares tienden a tener una fuerte retención y lealtad de los empleados; una sensación de buena voluntad acumulada con el tiempo porque están potencialmente más inclinadas a cuidar mejor a los empleados y ofrecer mejores beneficios que las empresas tradicionales no familiares.

- Debido a que muchas empresas familiares son fiscalmente conservadoras, la mayoría están en una buena posición para cuidar de sus empleados, incluso si eso significa que la familia propietaria puede necesitar suspender dividendos o ajustar la compensación.

- Además, debido a que las empresas familiares no están en deuda con inversores o con informes trimestrales de ganancias, pueden tomar decisiones financieras a largo plazo que pueden resultar en algunas pérdidas financieras a corto plazo, pero probablemente beneficiarán a la compañía a largo plazo, como retener a los mejores talentos.

- Esto ayudará a posicionar bien a la empresa para la reapertura de la economía. En este momento, estamos aconsejando a las empresas que piensen en los planes de contingencia de los empleados para los próximos seis, 18 e incluso 24 meses.

\section{Liquidez empresarial}

Basados en la postura de Koropp (2014), Encontrar estabilidad fiscal frente a un mercado tumultuoso debe seguir siendo el principal desafío al que se enfrentan muchas empresas familiares. Afortunadamente, las empresas familiares en particular tienden a ser fiscalmente conservadoras y la adversa a la deuda, lo que puede ayudar a limitar su exposición durante las recesiones económicas. Sin embargo, la falta de deuda por sí sola no significa inmunidad frente a los desafíos que pueden resultar en una reducción de la escala o un paro empresarial.

Hay varios pasos recomendados para que las empresas familiares tomen medidas para mantener aún más su liquidez, entre ellas:

- Aprovechando los aplazamientos del gobierno a las declaraciones de impuestos relacionadas con los ingresos y la nómina: 


\section{Revista científica, arbitrada e indizada, bajo la modalidad electrónica.}

Los Organismos gubernamentales encargados de recoger los impuestos, han permitido una extensión para las empresas y los individuos para 2020, que permite a las empresas preservar la liquidez. Muchas empresas familiares pueden aprovechar las extensiones de ingresos personales, ya que son entidades de flujo.

- Identificar qué pagos se pueden aplazar: Los líderes empresariales pueden examinar los presupuestos de flujo de efectivo y los planes para encontrar fuentes de capital que puedan liberar, o incluso aprovechar la liquidez familiar si es necesario. Muchas empresas familiares están aplazando las mejoras de capital y otros proyectos no considerados esenciales. Confirmar que está haciendo coincidir las entradas de efectivo con las salidas es fundamental para la gestión a lo largo de este período.

1. Solicitar una extensión o aplazamiento de los pagos de intereses de los bancos: Los desencadenantes de la Reserva Federal están ayudando a las empresas familiares a negociar intereses y términos para deudas y préstamos existentes, ayudando a aliviar las demandas de flujo de efectivo y están abriendo opciones para planificar necesidades inmediatas y a largo plazo. Las primeras indicaciones de los bancos es que están trabajando con sus clientes para ayudar a proporcionar ajustes a los acuerdos de préstamo.

\section{Liquidez familiar}

Si bien muchos propietarios de empresas familiares esperaban que esta interrupción fuera sólo unas pocas semanas, ahora están cambiando su pensamiento a escenarios a largo plazo. Como esta pandemia puede continuar durante varios meses, siguiendo los aportes de Cheltenham y otros (2017), las distribuciones de efectivo a los miembros de la familia pueden verse afectadas. Algunas empresas familiares han hecho planes para situaciones en las que el flujo de efectivo se vería interrumpido, incluyendo qué otros medios de flujo de efectivo podrían entrompetar la estabilidad financiera de las familias. En una abundancia de precaución, aconsejamos a los clientes que consideren el peor de los casos, que vemos en 


\section{Revista científica, arbitrada e indizada, bajo la modalidad electrónica.}

este momento como el mapeo de las necesidades de efectivo durante 24 meses para los miembros de la familia, suponiendo que no haya más dinero que venga del negocio.

Según El Kadi (2014), la transparencia en la comunicación de cualquier cambio, y la justificación de estos cambios, es esencial, ya que puede haber miembros de la familia en la junta o en la suite $\mathrm{C}$ de la empresa que pueda tener más acceso a la información financiera que otros miembros de la familia. Para superar cualquier brecha de conocimiento en temas tan sensibles, aconsejamos a los clientes que traten de mantener a todos en el redil, y explicar claramente cualquier decisión para cambiar dividendos. Aunque los argumentos no siempre se pueden evitar, una comunicación clara sobre la toma de decisiones debería ayudar a mitigar los conflictos, especialmente en tiempos ya estresantes.

Las implicaciones globales de COVID-19 en individuos y negocios están causando estrés y tensión en todas las áreas de la vida diaria. Los líderes de las empresas familiares se ven obligados a tomar decisiones empresariales ágiles para ayudar a proteger a los empleados y mantener la empresa a flote. A medida que las ramificaciones económicas de esta crisis siguen evolucionando, reconocer y abordar los desafíos lo más rápido y ágilmente posible puede ayudar a confirmar que las empresas familiares sobrevivirán al clima actual y posicionarse para recuperarse una vez que COVID-19 esté detrás de todos.

\section{MATERIALES Y MÉTODOS}

La investigación estuvo orientada por un enfoque postpositivista, cualitativo, de tipo documental, con diseño bibliográfico, comprendiendo la revisión literaria para conocer el estado del arte de las categorías estudiadas, al igual que el levantamiento de la información obtenida de las bases de datos, revistas científicas, trabajos de grado, repositorios institucionales, así como la identificación de objetivos. Sustentándose en los criterios expuestos por Pelekais et al., (2016), Pelekais et al., (2015) y Hernández et al., (2017, como un diseño interpretativo-crítico. 


\section{Revista científica, arbitrada e indizada, bajo la modalidad electrónica.}

\section{RESULTADOS}

Los hallazgos demuestran la existencia de gran cantidad de referente teórico sobre la temática, en la cual se desarrolla un aporte en la literatura de financiamiento de empresas familiares y presenta un modelo para guiar la investigación existente y futura mediante la identificación de brechas en las perspectivas teóricas y en elementos específicos del contexto, como la heterogeneidad de la empresa familiar, además de los factores específicos del país.

Estos resultados coinciden con lo expuesto por Chen et al., (2016), cuando refiere que la distancia entre las empresas familiares y los inversores externos es inmensamente grande, esencialmente por la llamada brecha de empatía entre propietarios e inversores o la retención generalmente preferida del control en lugar del crecimiento, al igual el desarrollo de la organización.

\section{CONCLUSIONES}

Una vez examinados los referentes teóricos y analizados los soportes documentales objeto de estudio, los hallazgos derivados de la correspondiente revisión reflejan la siguiente conclusión:

Motivada por la creciente atención a las empresas familiares en general, y sus decisiones de financiación en particular, esta revisión reúne los dos campos de investigación altamente relevantes de la empresa familiar y las finanzas. Como los conocimientos y contribuciones sobre las decisiones de financiación en las empresas familiares están bastante dispersos, se desarrolla un estado de la técnica en la literatura de financiación de empresas familiares y se presenta un modelo para guiar la investigación futura mediante la identificación de brechas en las perspectivas teóricas, el lado de la demanda frente a la oferta de financiamiento, y en todos los contextos. Más específicamente, se discute los argumentos subyacentes a las teorías tradicionales y profundizamos en su aplicabilidad para estudiar las decisiones de financiamiento en las empresas familiares. 


\section{Revista científica, arbitrada e indizada, bajo la modalidad electrónica.}

También se presentan oportunidades y desafíos de investigación futuras en todos los elementos específicos del contexto, como la heterogeneidad de las empresas familiares y los factores específicos de cada país. Se espera que esta revisión proporcione a los estudiosos de finanzas ideas de investigación fructíferas que eventualmente contribuirán a avanzar en la comprensión del comportamiento de financiamiento de empresas familiares en teoría y práctica.

\section{REFERENCIAS BIBLIOGRÁFICAS}

Cheltenham, E., Holt, D., Madison, K., \& Kellermanns, F. (2017). Variance in family members' assessments: The importance of dispersion modeling in family firm research. Family Business Review, 30, 61-83.

Chen, Q., Ding, S., Wu, Z., \& Yang, F. (2016). Family control, international accounting standards, and access to foreign banks: Evidence from international entrepreneurial firms. Journal of Small Business Management, 54, 598-621.

Chrisman, J. J., \& Holt, D. T. (2016). Beyond socioemotional wealth: Taking another step toward a theory of the family firm. Management Research, 14, 249-287.

Debicki, B. J., Kellermanns, F. W., Chrisman, J. J., Pearson, A. W., \& Spencer, B. A. (2016). Development of a socio-emotional wealth importance (SEWi) scale for family firm research. Journal of Family Business Strategy, 7, 47-57.

El Kadi, O. (2014) Autotomía caudal como estrategia gerencial para el cambio organizacional en empresas familiares. Tesis Doctoral. Universidad Privada Dr. Rafael Belloso Chacín. Venezuela.

Hernández, R., Fernández C., Baptista, P. (2017). Fundamentos de la investigación. Sexta edición. México: Editorial Mac Graw-Hill/Interamericana.

King, R., \& Peng, W. Q. (2013). The effect of industry characteristics on the control longevity of founding-family firms. Journal of Family Business Strategy, 4, 281-295

Koropp, C., Grichnik, D., \& Gygax, A. F. (2013b). Succession financing in family firms. Small Business Economics, 41, 315-334.

Koropp, C., Grichnik, D., \& Kellermanns, F. (2013). Financial attitudes in family firms: The moderating role of family commitment. Journal of Small Business Management, 51, 114-137.

Koropp, C., Kellermanns, F. W., Grichnik, D., \& Stanley, L. (2014). Financial decision making in family firms: An adaptation of the theory of planned behavior. Family Business Review, 27, 307-327

Martin, G., \& Gomez-Mejia, L. (2016). The relationship between socioemotional and financial wealth: Re-visiting family firm decision making. Management Research, $14,215-233$. 
Pelekais, C; Pertuz, F; Pelekais, E. (2016). Hacia una cultura de investigación cualitativa. Ediciones Astro Data S.A Maracaibo. Venezuela.

Pelekais, C; El Kadi, O; Seijo, C; Neuman, N. (2015). El ABC de la Investigación. Guía Didáctica. Ediciones Astro Data S.A. Maracaibo. Venezuela. 